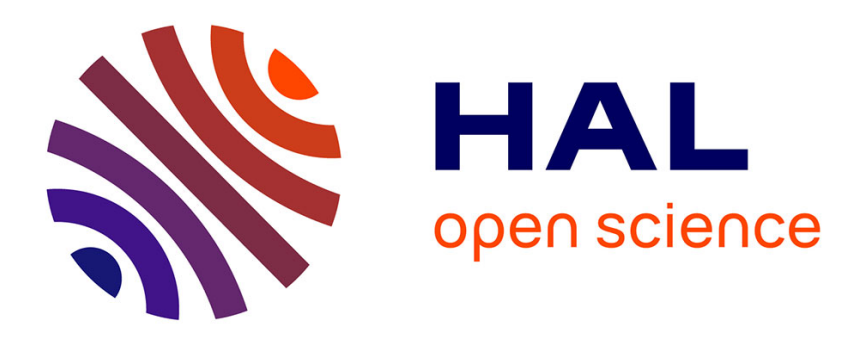

\title{
Sur les discontinuités de l'aimantation
}

Pierre Weiss, G. Ribaud

\section{To cite this version:}

Pierre Weiss, G. Ribaud. Sur les discontinuités de l'aimantation. J. Phys. Radium, 1922, 3 (3), pp.74-80. 10.1051/jphysrad:019220030307400 . jpa-00204308

\section{HAL Id: jpa-00204308 https://hal.science/jpa-00204308}

Submitted on 1 Jan 1922

HAL is a multi-disciplinary open access archive for the deposit and dissemination of scientific research documents, whether they are published or not. The documents may come from teaching and research institutions in France or abroad, or from public or private research centers.
L'archive ouverte pluridisciplinaire HAL, est destinée au dépôt et à la diffusion de documents scientifiques de niveau recherche, publiés ou non, émanant des établissements d'enseignement et de recherche français ou étrangers, des laboratoires publics ou privés. 


\section{SUR LES DISGONTINUITÉS DE L'AIMANTATION}

Par MM. Pierre WEIsS el G. RIBAUD

(Faculté des Sciences de Strasbourg.)

Barkhausen (') a montré en 1919 que, lorsqu'on soumet une substance ferromagnétique à un champ magnétique croissant ¿d'une manière continue, l'accroissement de l'aimantation présente des fluctuations d'allure accidentelle. /Ces fluctuations induisent dans une bobine entourant la substance un courant qui, par un amplificateur à lampes, peut ètre renforcé suffisamment pour iproduire dans isle téléphone un bruissement ou un crépitement caractéristiques. Le 'schéma ci-joint indique la disposition des appareils (Fig. 1).

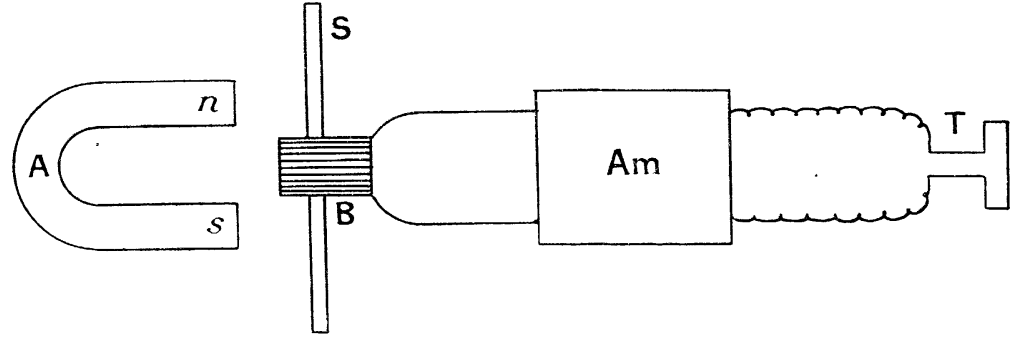

Fig.'. 1.

A, aimant; S, substance; B, bobine induile; Am. Amplificateur; T, téléphone.

Il suffit d'écouter au téléphone T, pendant qu'on approche ou qu'on éloigne le petit aimant $\mathrm{A}$ de la subtance $\mathrm{S}$.

Ce phénomème a fait l'objet d'une étude intéressante 'de M. B. van der Pol Jr. $\left({ }^{2}\right)$.

M. van der Pol a trouvé que le phénomène se présente dans toutes les substances ferromagnétiques examinées, avec des intensités d'ailleurs inégales. Dans certaines d'entre elles il pouvait ètre perçu sans l'emploi de l'amplificateur. Il était particulièrement intense dans un fil de ferronickel, de la leneur donnant une dilatation égale à celle du platine.

M. van der Pol a montré que le phénomène ne se produit d'une manière marquée que dans les régions de variation rapide $\mathbf{A} \mathbf{B}, \mathbf{C} \mathbf{D}$ du cycle d'aimantation (Fig. 2).

Il a pu faire une évaluation du "nombre "des discontinuités dans un cycle et montrer ainsi que la quantité de matière intéressée dans l'une

(1) Phys. Zeitschr., t. 20 (1919) p. 401.

$\left.{ }^{2}\right)$ Acad. Amsterdam, t. 23 (1920), pp. 637 et 980 . 
d'entre elles est faible. Elle a élé trouvée dans deux cas de l'ordre du cinquième de millimitre cube. Par l'emploi simultané de deux bobines induiles, placées à une dislance variable sur le fil et l'observation des courants induits dans deux galvanomètres, il a pu établir que jusqu'ì une distance de 7 centimètres les impulsions communiquées aux galvanomètres étaient souvent synchrour. II en résulle que. dans certains cas, les régions subissant la mème varialion brusque de l'aimantation ont, malgré leur laible volume, un grand développement en longueur ; er sont probablement des cristaux étirés par le laminage.

M. van der Pol a remarqué que les mèmes discontinuités se produisent lorsqu'une aimantation rémanente est détruite par des tractions répétées, phénomène qui cesse lorsque Jaimantation

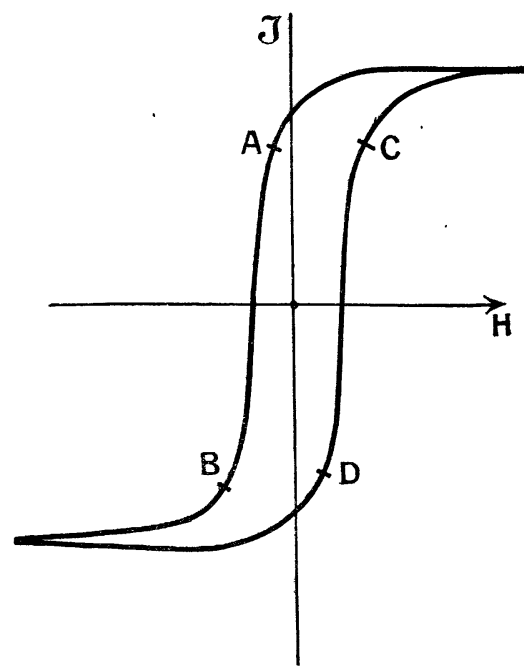

Fig. ‥ rémanente a disparu. La lorsion donne lieu à des observations analogues; de même, la destruction du magnélisme rémanent par la chaleur.

La flexion est particulièrement intéressante. Quand un fil de fer a reçu une cerlaine aimanlation rémanente, celle-ci peut ètre détruite avec accompagnement de sons caractéristiques par des flexions données alternativement en sens contraires. Mais mème après. si l'on continue les flexions, on entend les sons, mais un peu plus faiblement. Un fil récemment recuit. qui n’a jamais élé aimantí. présente le même phénomène. La flexion peut être continuéc indéfiniment et le son persiste avec la mème intensité. Cette expérience. ne peut guère ètre interprétée que 'comme une manifestation de l'existence de l'aimantation spontanée donnée par la théorie du champ moléculaire. Le phénomìne n'est pas imputable au champ magnétique terrestre. On peut compenser ou renforcer ce dernier sans altérer en aucune façon le phénomène.

Enfin M. van der Pol a pu constater sur fle fil ,de ferronickel que les sons révélateurs des discontinuités se prolongeaient pendant plusieurs secondes après la variation du champ. C'est le phénomène ‘connu sous le nom de viscosité magnétique.

Nous avons répété quelques-unes de ces expériences sous une đorme permettant de les présenter à un nombreux auditoire et nous avons noté à 
cetle occasion quelques particularités nouvelles. Nous nous sommes servi à cel effet de deux amplificateurs à basse fréquence à 3 lampes, mis en série, et nous les avons reliés à une paire de téléphones rendus haut parleurs par l'addition de cornets acoustiques. Les phénomènes peuvent facilement être perçus par 200 personnes.

On sait, par l'étudede l'aimantation dans les champs faibles, que l'effet du champ peut ètre séparé en une partie réversible et une partie irréversible qui obéissent à des lois de varialion thermique différentes. Considérons l'expression

$$
J=a H+b H^{2}
$$

où $\mathfrak{J}$ est l'intensité d'aimantation; $\boldsymbol{H}$, le champ magnétique: $a, b$, des constantes (à une température déterminée); cette formule représente te lieu des sommets des cycles de petite amplitude, le premier terme mesure la partie réversible et le second la partie irréversible du phénomène.

Dans les cycles de grande amplitude on sait que les variations rapides sont liées au développement des phénomènes irréversibles. On sait d'autre part que les substançes magnétiques usuelles, homogènes en apparence, sont composées d'innombrables petits cristaux de propriétés magnétiques anisotropes. La simplicité des phénomènes élémentaires

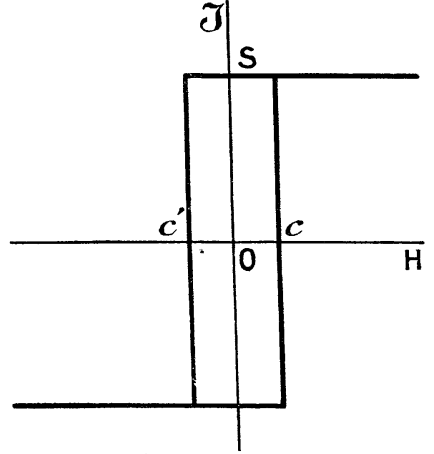

Fig. 3 . propres à chaque cristal, si elle existe, ne peut qu'ètre estompée dans l'effet de moyenne que donne l'observation. Il était donc naturel de se demander si les phénomènes déjà extrèmement brusques que donne l'observation sur les substances à cristallisation confuse ne seraient pas en réalité infiniment brusques, c'est-à-dire si à une variation infiniment petite du champ ne correspondrait pas une variation finie de l'aimantation. I'étude des propriétés du cristal de pyrrhotine, l'étude faite par Maurain des dépòts de fer électrolytique obtenus dans le champ magnétique, deux cas où la substance possède des propriétés uniformes, sont favorables à cette schématisation. Voici comment on peut se représenter le phénomène dans une matière réellement homogène.

Il existe dans une semblable matière une direction de facile aimantation. Le cycle d'aimantation, pris dans cette direction, est rectangulaire (fig. 3); sa hauteur est égale à deux fois l'aimantation à satu- 
ration OS; sa largeur, à deux fois un champ coercitif caractéristique $0 \mathrm{C}=\boldsymbol{H}_{c}$. L'aimantation est entièrement irréversible.

Supposons maintenant qu'on aimante la mème substance au mojen d'un champ incliné sur la direction de facile aimantation. La figure ci-jointe (fig. 4) montre comment le recteur constant : aimantation à saluration (ou aimantatioli spontanée) se déplace dans le plan $x$ OH contenant la direction de lacile aimantation $0 x$ et celle du champ $\mathrm{OH}$.

Si nous complons l'aimantation en prenant seulement sa



Fig. 4 .

composante dans la direction du champ, le cycle aura l'aspect ci-contre

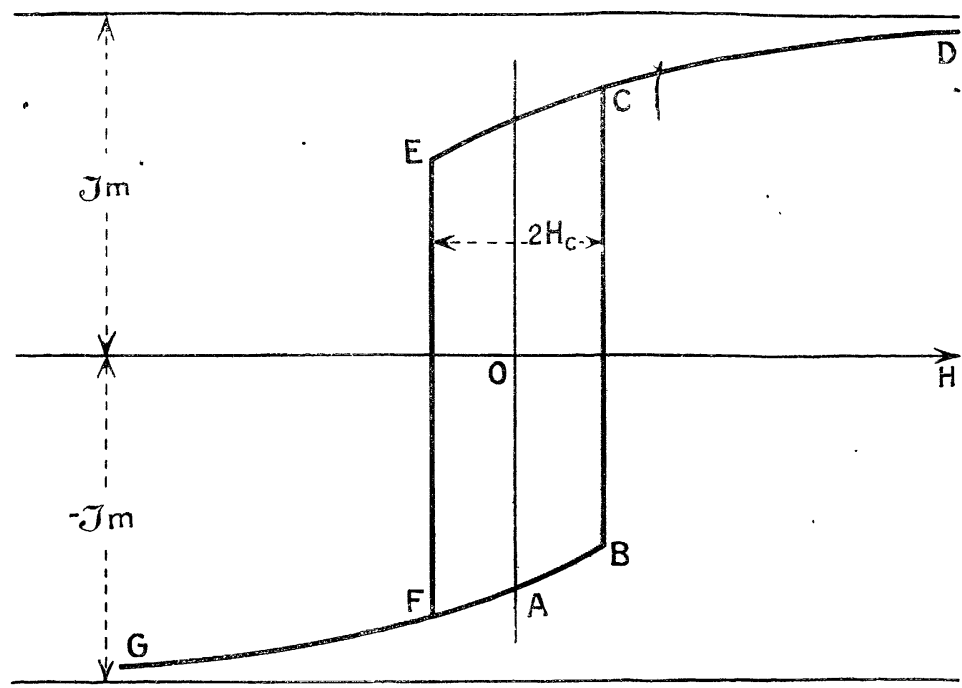

Fig.

(fig. 5). (Les lettres se correspondent dans les deux figures). Partons du 
point A. Laimantation y est égale à l'aimantation à saturation mais diminuée par son obliquilé sur le champ. Elle est négative. si nous laisons agir un champ posilif, l'obliquité augmente d'aborl par rotation réversible de l'aimantation, et cela jusqu'au moment où la composante du champ tans la direction de l'aimantalion atteindra le champ coercitif (point B). Le relournement brusque de l'aimantation se produit, l'aimantalion varie de $B$ à $C$ sans que le champ varie en mème temps.

Puis, le champ continuant à croìtre, l'aimantation lourne d'une maniere réversible en tendant à devenir parallèle au champ. Lorsque le champ décroìt, l'aimanlation décrit DE par rotation réversible, passe sous l'influence de $\boldsymbol{I}_{c}$ de $\mathrm{E}$ à $\mathrm{F}$ d'une manière irréversible et ainsi de suite.

Ici les phénomènes réversible et irréversible existent denc tous deux, mais leur mécanisme est différent. Dans les deux cas que nous venons d'examiner, le phénomène irréversible a la brusquerie nécessaire pour produire par induction un courant réagissant au téléphone.

Il est clair que si l'on forme une matière isotrope au moyen de cristaux élémentaires possédant ces propriélés schématiques, on réalisera un corps ayant des propriétés voisines de celles des substances réelles. Le retournement brusque de l'aimanlation se produira pour des champs $\frac{/ I_{c}}{\cos z}$, où $H_{c}$ est le champ coercitif du cristal élémentaire, avec accumulation, comme il est facile de s'en rendre compte. aux valeurs de $\cos \alpha$ peu éloignées de l'unité. Les directions de facile aimantation très inclinées sur le champ donneront lieu au contraire à des phénomènes ne se produisant que pour des champs élevés et de plus en plus rares pour un invervalle de chanp donné ( $\left.{ }^{1}\right)$.

Les portions de la courbe expérimenlale voisines de la saluration sont donc données par cette synthèse comme' étant réversibles. L'expéricnce confirme cette manière de voir. Elles doivenl, pour la mème raison, itre dépourvues de variations brusques. C'est ce que van der Pol a indiqué par los limites qu'ıl a données pour les régions des cycles présentant des discontinuités.

Voici maintenant quelques observalions.

Magnétite. - Le bruil produit par les discontinuités a été particulièremenl fort avec une plaque de magnétite de 7 centimètres de long, laillée parallèlement au dodécaèdre dans un beau cristal de Brozzo.

(1) Les actions mutuelles entre les crislaux élémentaires diversement aimantés doivent contribuer à estomper encore les phénomènes. 
Cela surprent un peu dans le cas d'un cristal où l'on pouvait s'atteudre à des phénomèmes unilormes comme pour la pyrrhotine. Mais cela s'accorde fort bien avec l'étude directe de l'aimantation des cristaux de magnétite, qui montre que le cristal n'a qu'en apparence la symétrie cubique. Les phénomènes magnétiques ne satisfont qu’avec une grossière approximation à cette symétrie, comme si elle résultait d'une compensation imparfaite de phénomènes de symétrie moindre provenant de cristaux élémentaires diversement orientés.

L'observation des discontinuités nous apprend que le grain de la structure est probablement assez gros.

Nous avons essayé aussi une magnétite compacte, qui donne des effets faibles, et dont la structure est sans doute très différente.

Fer doux. Invar. - Avec des tiges, ou des fils de ces deux substances, l'observation est très facile. Il est particulièrement intéressant de constater que lorsqu'on décrit la bande descendante $A B$ du cycle (fig. 6) et qu'on la quitte suivant BC en faisant décroitre le champ négatif, on n'a aucun bruit le long de la portion à peu près réversible $\mathrm{BC}$. aussi bien à l'aller qu'au retour en revenant de $\mathrm{C}$ en $\mathrm{B}$, où l'on rejoint de nouveau la branche descendante. Puis, brusquement, lorsqu'on repasse par $\mathrm{B}$, le bruit reprend avec sa pleine intensité. G'est comme si, par la descente $\mathrm{AB}$, on avait “ effacé » le phénomène

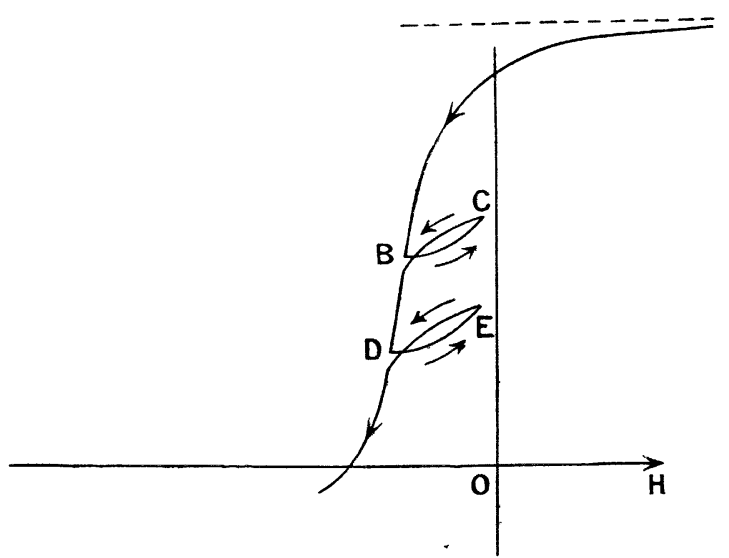

Fig. 6. jusqu'en $\mathbf{B}$, et comme si au delà de $\mathbf{B}$ vers $\mathbf{D}$ la réserve en était restée intacte. On peut répéter cette expérience, [représentée par la boucle BC, plusieurs fois sur la branche descendante avec le mème succès. Si par exemple à partir de $\mathrm{D}$ on diminue le champ, c'est le point $\mathrm{D}$ qui joue le ròle de limite de la réserve de bruit.

On peut réaliser cette expérience en produisant les champs démagnétisants au moyen d'un barreau aimanté que l'on approche de la substance. Les points B, D, etc. correspondent des positions $B^{\prime}, D^{\prime}$ de l'extrémité de l'aimant (fig, 7). 
L'expérience, sous cette forme, éveille l'idée que l'aimant dans son avance balaie la réserve de bruit jusqu'au point le plus rapproché de la

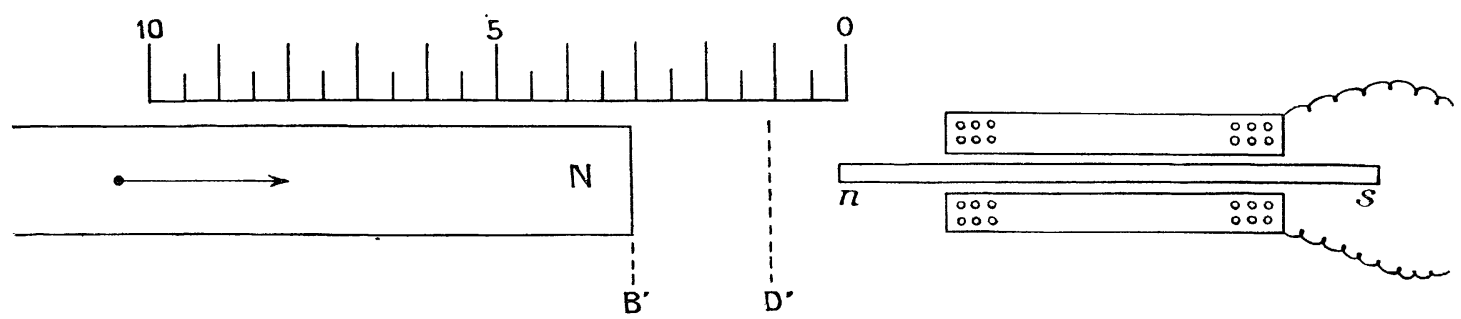

Fig. 7 .

substance qu'il a atteint. Les mourements qu'on lui donne en deçà de cetle limite ne produisent aucun effet.

Champ terrestre. - Avec la magnétite de l’expérience ci-dessus les effets sont assez intenses pour que l'on puisse faire entendre à un nombreux auditoire l'effet du champ terrestre. On place la magnétite dans une bobine induite dont l'axe coïncide avec la direction du champ magnélique terrestre et avec la plus grande dimension de l'échantillon. On aimante cette magnétite avec un aimant en acier dans la direction du champ terrestre, puis après avoir écarté l'aimant on la retourne bout pour bout, avec la bobine l'entourant, de façon que le champ terrestre intervienne comme champ démagnétisant. On entend le bruissement caractéristique.

Si l'aimantation initiale de la magnétite est dirigée en sens inverse du champ terrestre le retournement de la bobine est sans effet, le champ terrestre intervenant alors pour renforcer l'aimantation.

Viscosité magnétique. - Elle est facile à observer avec un tore, qui a été aimanté fortement dans le sens positif, par exemple, et que l'on soumet brusquement à un champ négatif. Quand ce dernier est voisin du champ coercitif, la viscosité se manifeste par le bruissement dù aux discontinuités, qui continue arec une grande intensité pendant un temps assez long (jusqu'à 14 secondes). 